\title{
Compact duplexer with miniaturized dual loop resonator
}

\author{
C. Collado ${ }^{1}$, J. Pozo $^{1}$, J.Mateu ${ }^{2}$, J.M. O’Callaghan ${ }^{1}$ \\ ${ }^{1}$ Universitat Politècnica de Catalunya (UPC). Departament de Teoria del Senyal. Campus Nord UPC D3. C/Jordi \\ Girona 1. Barcelona, 08034 Spain Phone :+34934017229. \\ ${ }^{2}$ Centre Tecnològic de Telecomunicacions de Catalunya (CTTC). Building Nexus I, Gran Capità, 2-4. $3^{\text {rd }}$ floor. \\ Barcelona, 08034 Spain Phone :+34932058046.
}

\begin{abstract}
This work presents a very compact duplexer based on novel miniaturized loaded-close-loop dual resonators. Two different dual resonators are used to establish the two bands, corresponding to the transmitter and the receiver bands. The two filters are connected to the antenna through a matching network. We have fabricated a prototype using a $\mathbf{1 0 . 2}$ dielectric constant substrate. The duplexer fits in a $16 \times 10 \mathrm{~mm}^{2}$ area and has less than $2 \mathrm{~dB}$ insertion loss and more than $20 \mathrm{~dB}$ of isolation. Measurement results are in good agreement with the simulation.
\end{abstract}

\section{INTRODUCTION}

Constant development in wireless communication systems has created the need of compact, high performance and cost effective microwave and RF components [1]. Planar technology offers a convenient way to realize miniature and cost effective devices since their fabrication is simple and reliable. On the other hand, the advance in computing speed has allowed to design very complex planar structures by means of using powerful electromagnetic simulators.

This paper presents a very compact duplexer to route the signal from the transmitter to the antenna and from the antenna to the receiver [2]. This element is needed in mobile terminals of cellular communications using standards that require simultaneous transmission and reception [3]. In our design the duplexer consists of two band pass filters connected to the antenna through a matching network. The compactness of the duplexer is achieved by using a novel miniaturized dual resonator, which performs as a two pole band pass filter. The dual resonator consists of a square-shaped close loop loaded with open ended stubs. The stubs allow to set the central frequency and bandwidth of the filters.

The design has been simulated and optimized using a full-wave electromagnetic simulator (Momentum). As detail later, there is good agreement between measurements and simulations.

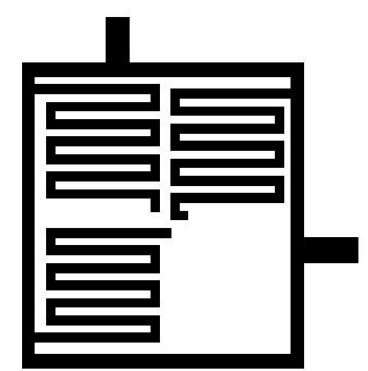

a)

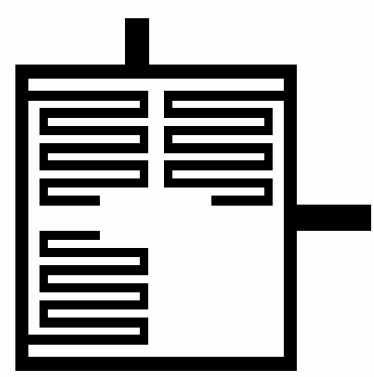

b)
Figure 1: Outline of the two dual resonators. a) upper band (transmitter band) b) lower band (receiver band).

\section{DUAL RESONATOR}

As pointed out in the introduction the dual resonator consists in a square-shaped close loop resonator loaded with open-ended stubs. The purpose of loading the loop is twofold: 1) to shift down the resonant frequency of the original resonator, that is to get a very small structure and 2) to break the degeneracy between the two resonant modes that would exist in closed rectangular resonator. Figure 1 shows the two dual mode resonators that form the bandpass of the filters. Note that both resonators differ on the length of the stubs. This is because they correspond to different pass bands. The resonator outlined in figure 1a corresponds to the lower band

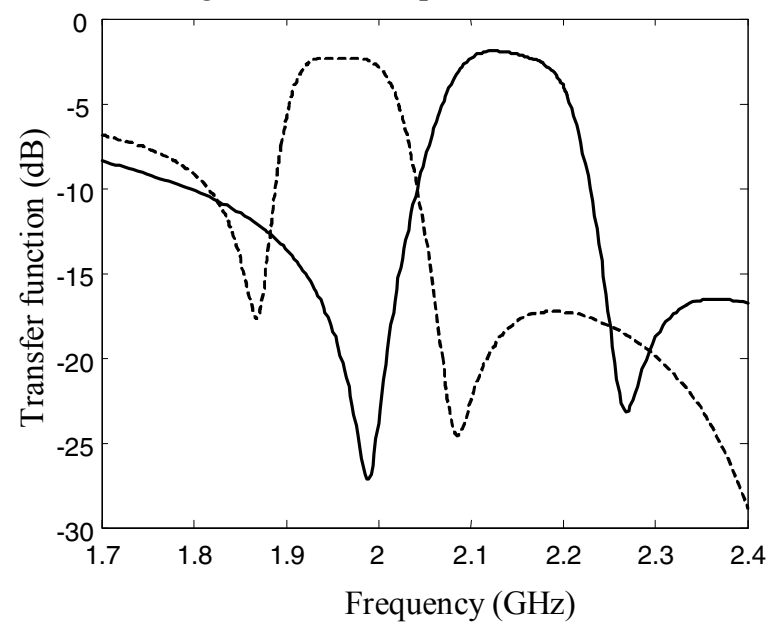

Figure 2: Transfer function of the filter of figure 1a (solid line) and figure $1 \mathrm{~b}$ (dashed line). 
(transmitter band) whereas the resonator outlined in figure $1 \mathrm{~b}$ corresponds to the higher band (receiver band). The simulations have shown that the degree of miniaturization also depends on the place where the stubs are connected. The bandwidths of the filters depend on the coupling between the two resonant modes of the resonator. The coupling is easily adjusted by fitting the length of the open-ended stubs.

Another highly important issue in the filter design is the external coupling, that is the way in which the input and output ports of the filter are coupled to the filter resonator. In this case, we have chosen a direct contact coupling. These couplings usually define the ripple of the passband and are adjusted by fitting the connecting point of the $50 \mathrm{ohm}$ feed lines.

The two filters of figure 1 have been designed and simulated with a full-wave electromagnetic simulator (Momentum). We have used a substrate with 10.2 dielectric constant and $0.635 \mathrm{~mm}$ thickness. The metal thickness is $35 \mu \mathrm{m}$. The square loop fits into a $6.4 \times 6.4$ $\mathrm{mm}^{2}$ area. The width of the external loop line, $d$, is $0.3 \mathrm{~mm}$ and the width of the inner stubs, $g$, is $0.2 \mathrm{~mm}$. Figure 2 shows the simulated transfer function of those designs. The designed filters exhibit $2 \mathrm{~dB}$ insertion loss and sharp frequency roll-off.

\section{MATCHING NETWORK}

The aim of the matching network is to connect the two filters (the one of the transmitter side and the one of the receiver side) to the same antenna ensuring good isolation between the transceiver and the receiver.

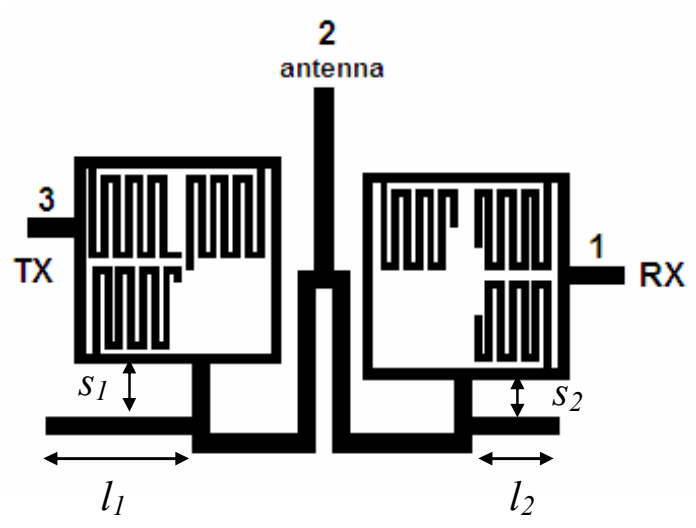

Figure 3: Design of the duplexer.

In our approach we connect the filters as shown in figure 3. Port 1 represents the output of the receiver filter, port 3 represents the input to the transmitter filter and the antenna is represented by port 2 . All ports match to 50 ohms and the matching network has been designed using $50 \mathrm{ohms}$ transmission lines (width of $0.57 \mathrm{~mm}$ ). This matching network consists in a transmission line which connects the two filters with the antenna port connected in the middle. The meander line is loaded with two stubs, as shown in figure 3 , whose lengths $\left(l_{1}\right.$ and $\left.l_{2}\right)$ and connection points $\left(s_{1}\right.$ and $\left.s_{2}\right)$ are very critical for the duplexer performance.

The design has consisted in adjusting $l_{1}, l_{2}, s_{1}$ and $s_{2}$ to guarantee the requirements of the duplexer. Next section

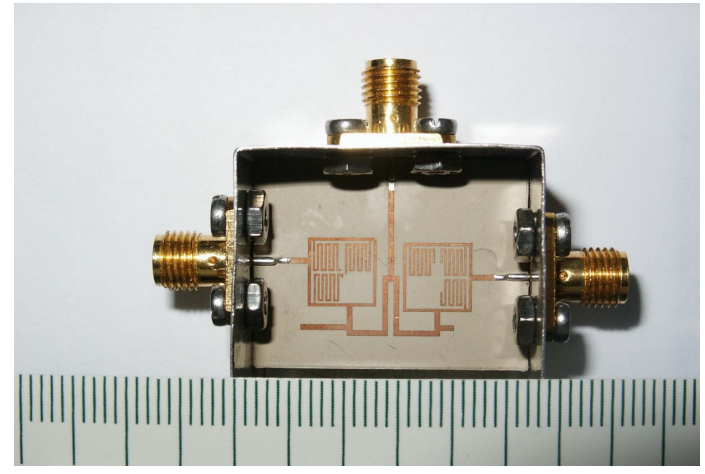

Figure 4: Picture of the microstrip duplexer. The scale is in $\mathrm{cm}$.

describes the design, simulation and shows the measurements of the implemented duplexer.

\section{DUPLEXER SIMULATION AND RESULTS}

The duplexer designed and fabricated is the one shown in figure 4. The dimensions of the filters are the ones mentioned in section II. The parameters which defined the matching network $\left(l_{l}, l_{2}, s_{1}\right.$ and $\left.s_{2}\right)$ are $4 \mathrm{~mm}, 1.2 \mathrm{~mm}$, $1.9 \mathrm{~mm}$ and $1.35 \mathrm{~mm}$, respectively. The whole duplexer fits in a $16 \times 10 \mathrm{~mm}^{2}$ area. The device has been fabricated using a 10.2 dielectric constant substrate with $0.635 \mathrm{~mm}$ thickness and with a $35 \mu \mathrm{m}$ metallization thickness.

The duplexer was measured with a E8361A network analyzer from Agilent Technologies. Figure 5a depicts the simulated and measured isolation between the transmitter and receiver, i.e. $S_{13}$ scattering parameter. Figure $5 \mathrm{~b}$ shows the simulated and measured transmitter parameters, that is the signal route from the antenna to the receiver, $S_{12}$, and the signal route from the transmitter to the antenna, $S_{23}$.

The results illustrate very good agreement between measurements and simulation. The isolation between the transmitter and the receiver is better than $20 \mathrm{~dB}$ and the insertion loss in the receiver and transmitter band are better than $2 \mathrm{~dB}$. By comparing this duplexer with a commercial one [4], we find that the insertion losses are comparable, but the isolation of the commercial duplexer is considerably higher. On the other hand, simplicity (and probably cost) are advantages of the design presented here.

\section{CONCLUSIONS}

We have designed and fabricated a very compact duplexer based on two dual resonators and a matching network. The dual resonators consist in a square-shaped close loop resonator loaded with open-ended stubs. The bands and central frequency of the resonator have been chosen by adjusting the loading stubs. This device was fabricated in a microstrip technology which offers a very cost effective device. The duplexer has been designed and fabricated. The measured results show a good agreement with the simulation. 

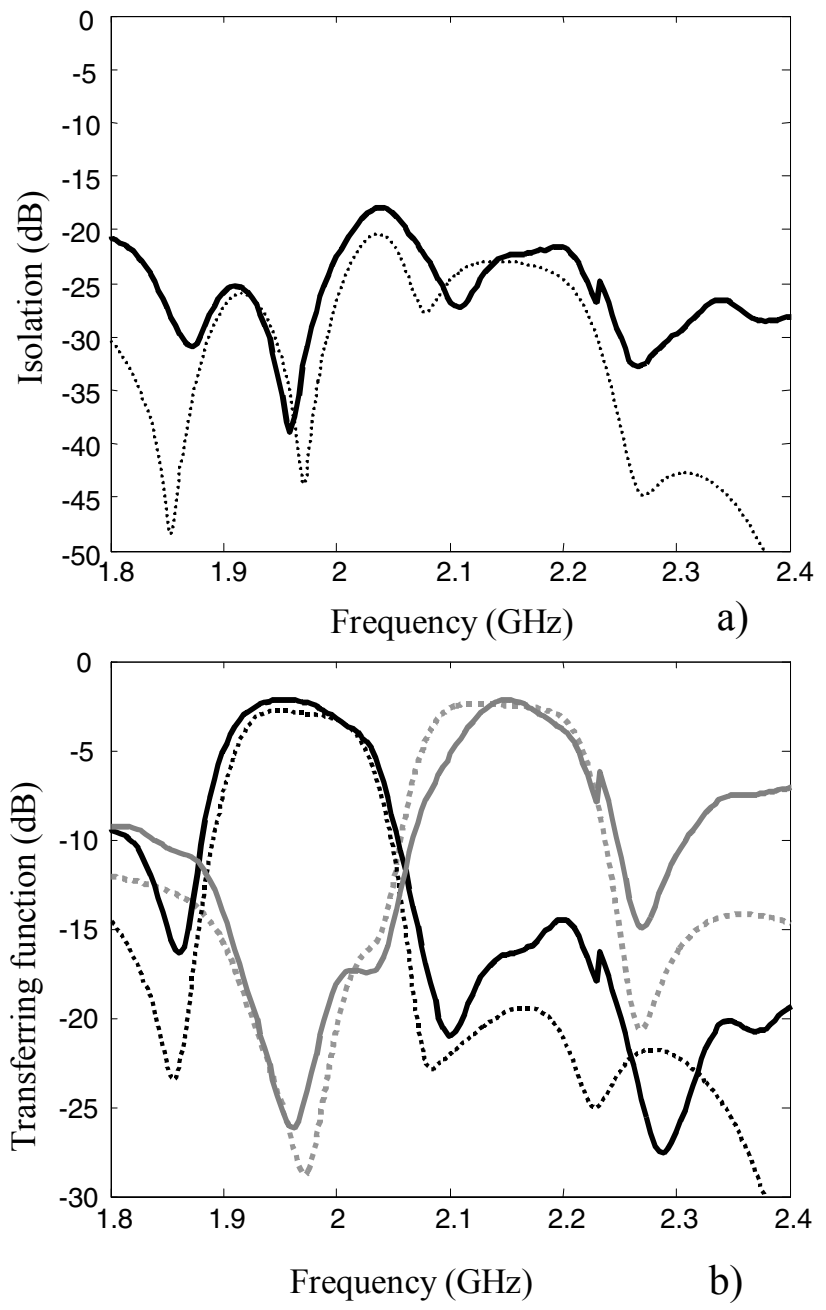

Figure 5: a) Simulation (dotted line) and measurement (solid line) isolation between transmitter and receiver. b) Simulation (dotted line) and measurement (solid line) insertion loss from transmitter to antenna (black) and from antenna to receiver (grey).

\section{REFERENCES}

[1] R. Levy, R.V. Synder, G. Matthaei, "Design of Microwave Filters" IEEE Trans. Microwave Theory and Techniques, vol. 50. no3, pp 783-793, March 2002.

[2] D. M. Pozar, Microwave Engineering, John Wiley \& Sons 1998.

[3] J.S. Hong, M.J. Lancaster, Microstrip filters for RF/Microwave applications, John Wiley \& Sons, 2001.

[4] Duplexer for W-CDMA, Ube Electronics, P/N UX1950L207. 
